\title{
Identification of phylogenetically conserved sequence motifs in microRNA 5' flanking sites from $C$. elegans and $C$. briggsae Liisa Heikkinen ${ }^{1,2}$, Suvi Asikainen ${ }^{1,2}$ and Garry Wong*1,2
}

\author{
Address: ${ }^{1}$ Department of Biosciences, University of Kuopio, PO Box 1627, Kuopio 70211, Finland and ${ }^{2}$ Department of Neurobiology, AI Virtanen \\ Institute, University of Kuopio, PO Box 1627, Kuopio 70211, Finland \\ Email: Liisa Heikkinen - liisa.heikkinen@uku.fi; Suvi Asikainen - suvi.asikainen@uku.fi; Garry Wong* - garry.wong@uku.fi \\ * Corresponding author \\ Published: 26 November 2008 \\ BMC Molecular Biology 2008, 9:105 doi:10.1 186/147/-2199-9-105 \\ This article is available from: http://www.biomedcentral.com/147I-2199/9/105 \\ (C) 2008 Heikkinen et al; licensee BioMed Central Ltd. \\ This is an Open Access article distributed under the terms of the Creative Commons Attribution License (http://creativecommons.org/licenses/by/2.0), \\ which permits unrestricted use, distribution, and reproduction in any medium, provided the original work is properly cited. \\ Received: 2 June 2008 \\ Accepted: 26 November 2008
}

\begin{abstract}
Background: MicroRNAs (miRNAs) are small, noncoding RNA molecules that act as posttranscriptional regulators of gene expression. Studies concerning transcriptional regulation of miRNAs have so far concentrated on those located within the intergenic region of the genome and the search for putative promoters, thus leaving open the question of the existence of possible regulatory elements common to all miRNAs including those located in introns of protein coding genes.
\end{abstract}

Results: In this study, we initially searched for motifs occurring in the area 1000 bp upstream from all miRNAs independent of their genomic location. We discovered a previously unknown sequence motif GANNNNGA that displayed a conserved distribution in the nematode worms Caenorhabditis elegans and Caenorhabditis briggsae. This motif had a peak occurrence at 500 bp upstream, with a sharp drop-off toward the miRNA start site. Further analysis indicated that this motif was locally restricted and not enriched 1000-5000 bp upstream or 0-2000 bp downstream of the miRNA start site. In addition, this motif was observed to be most abundant in the upstream sequences of two important miRNAs, mir-I and mir-I24. This abundance was also conserved in phylogenetically distant species including human and mouse.

Conclusion: The results show that the motif GANNNNGA is conserved close to miRNA precursor start sites, suggesting that it may be involved in miRNA sequence recognition or regulation. This data provides important knowledge for the identification and computational prediction of miRNA sequences.

\section{Background}

MicroRNAs (miRNAs) form one class of small, non-protein coding RNAs. They are defined as single stranded, 19$25 \mathrm{nt}$ long endogenously expressed RNAs generated from one arm of a hairpin precursor sequence [1-3]. These precursors are derived from a larger transcript, pri-miRNA, which is considered to be the original transcription product of a miRNA gene. MiRNAs are known to be involved in the post-transcriptional regulation of protein-coding genes. They operate by binding to the 3'UTR region of a mRNA sequence with antisense base pairing and cleave the target mRNA or repress its translation into protein. One miRNA can repress various different mRNAs and a single mRNA may be bound by several co-operating miRNAs $[4,5]$. The expression of a miRNA may be associated with different developmental stages of an organism or, 
more often, they are found to be expressed in particular cell types $[2,3]$.

The transcriptional regulation of miRNA genes is still not completely understood. Most miRNA genes are located in the genome far away from protein coding genes and are therefore thought to derive from their own, independent transcription units [2,3]. There is also some evidence that these "intergenic" miRNAs are transcribed from their own promoters by RNA Polymerase II $[6,7]$, and that a small fraction of miRNAs interspersed among the Alu repeats in human genome may be transcribed by RNA Polymerase III [8]. MiRNAs organized to clusters are also suggested to be transcribed independently as multi-cistronic units $[9,10]$. Those miRNAs whose origins are in introns of protein coding genes are possibly transcribed either in parallel with their host genes or independently $[2,3]$.

The upstream regions of intergenic miRNAs have been inspected in several studies to reveal additional information about miRNA transcription [7,11-13]. In these studies, some interesting sequence motifs have been found and also a few known transcription factors are suggested to regulate the miRNAs. The focus has thus far mainly been in the putative promoters of miRNA genes that are located in the intergenic area, thus leaving open the question of possible regulatory elements of all the other miRNAs.

In this study, we examined the upstream sequences of Caenorhabditis elegans and Caenorhabditis briggsae miRNAs in order to find over-represented, phylogenetically conserved short sequence motifs that occur upstream from miRNAs, independent of their origin in the genome. In the analysis, we used four motif finding tools with different algorithms and motif models. The most significant motif found was GANNNNGA, a novel motif with conserved distribution upstream of the miRNAs in these worms. This motif was also found to be especially abundant in the upstream sequences of two old and biologically important miRNAs, mir-1 and mir-124, thus suggesting a connection between the number of motif instances in the upstream sequence close to a miRNA start site and a globally conserved function of the miRNA.

\section{Results}

The main workflow of the study is shown in Figure 1.

\section{Search for conserved motifs}

The putative miRNA promoter sequence sets, containing the sequences up to $1000 \mathrm{bp}$ upstream from the starting site of 100 Caenorhabditis elegans miRNAs (25 of these were located within a protein coding gene) and 95 Caenorhabditis briggsae miRNAs, were searched for conserved over-represented sequence patterns with four motif finding tools: POCO, Weeder, MEME and Gibbs Motif Sampler.

With POCO, 65 significant patterns that occur in every sequence in both $C$. elegans and $C$. briggsae putative miRNA promoter sequence sets were found (Table 1). The found patterns were 4-8 bp long, containing four known nucleotides A, C, G or T while the rest of the places in a pattern, marked as $\mathrm{N}$, can be any of these. There were four patterns having an average of more than 10 occurrences in both of our putative miRNA regulatory region data sets: GAGA (11.6 in C. elegans/15.4 in C. briggsae), GANNNNGA (11.3/14.8), GANANNG (11.0/13.1) and ANAGNG (10.9/11.0), thus containing abundant A and G. To check if these nucleotides are dominating in our putative regulatory sequences, the proportion of all nucleotides was calculated from all the sequences of C. elegans and $C$. briggsae. The most frequent nucleotides were A (30.9\% in C. elegans and $29.9 \%$ in C. briggsae) and $\mathrm{T}$ (30.8\% and $29.7 \%$, respectively), while both C and G had about $20 \%$ proportions. The multiple sequence alignment of the over-represented patterns common to both data sets gave for consensus sequence GANNNNGNG, a pattern that closely resembles GANNNNGA, one of the most copious patterns found from both putative miRNA regulatory sequence sets. Thus, from all the POCO results, we selected GANNNNGA for further study. In addition, to find if this actually was a repetitive motif, we counted also the occurrences of GANNNNGANNNNGA and found it from 57 C. elegans and 58 C. briggsae putative miRNA regulatory sequences.

Both putative miRNA regulatory sequence sets were further scanned for over-represented short patterns using three motif finding tools with different algorithms and motif models. With Weeder, the most significant pattern found was GAAGGAGG, located 473 times from 94 C. elegans and 623 times from 93 C. briggsae miRNA upstream sequences. Further, with Gibbs Motif Sampler, the most frequent motif revealed was TTTCAAAAA, with 1218 instances in 98 C. elegans sequences, but only 623 instances in 94 C. briggsae sequences. The two most significant patterns found with MEME had consensus sequences CTCCGCCC and TTTCAAAA, the latter of which is a more well-defined version of the motif with similar consensus found with Gibbs Motif Sampler. These two motifs were found only from 57 and 63 C. elegans miRNA upstream sequences, respectively. All the motifs found with different tools together with the motif sequence logos and the motif $p$-values calculated against random background are gathered to Figure 2 .

\section{Motif analysis}

The Transfac 7.0 public database [14] contains only a few nematode transcription factor binding sites, and none of them could be matched with any motif we had found. 


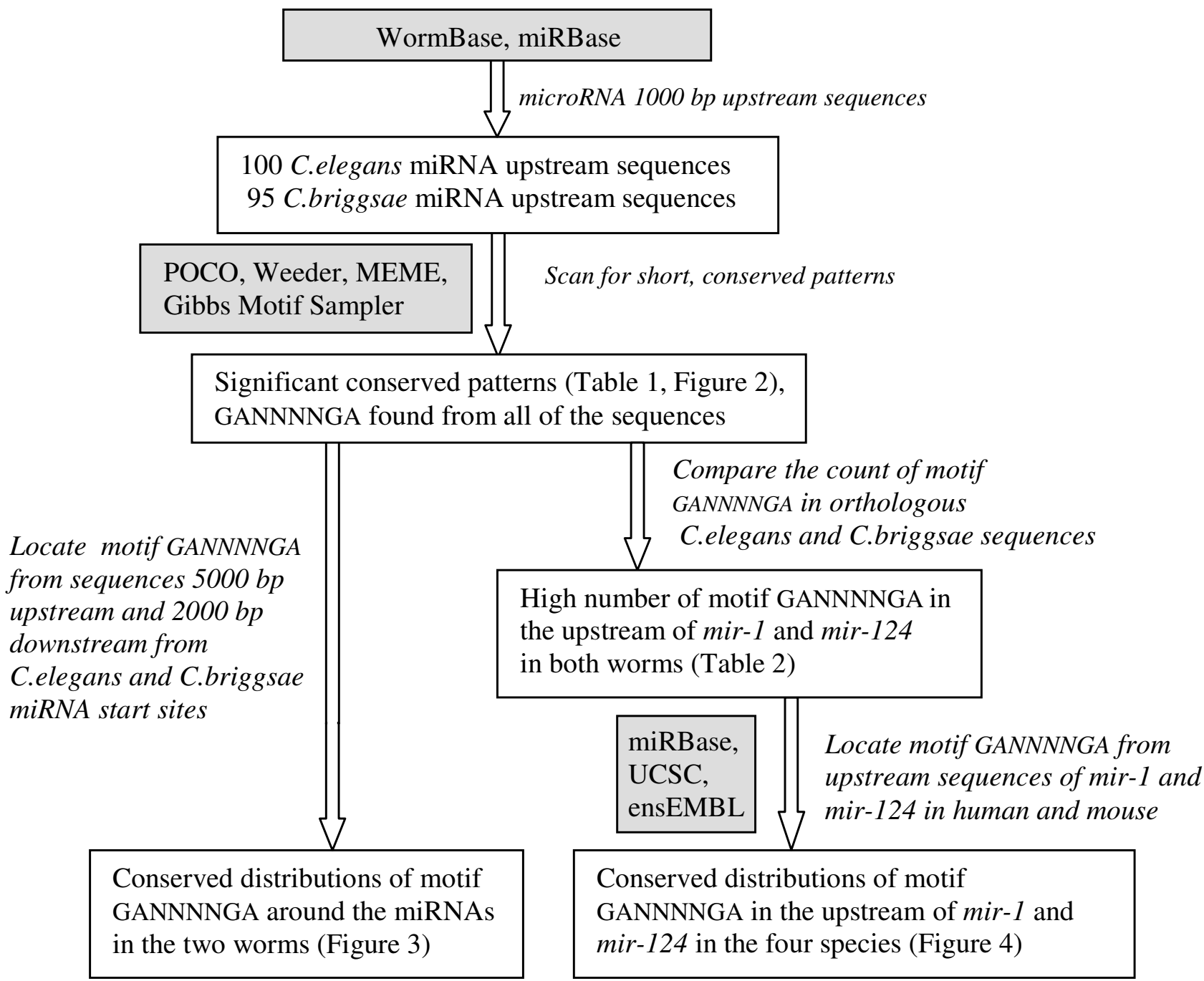

Figure I

The main workflow of this study.

We did find a human match with ISGF-3 from Transfac, and STAT1 from the JASPAR v3 database [15]. Some of these motifs we observed in C. elegans were reported in earlier studies concerning C. elegans intergenic miRNA promoter elements. The motif CTCCGCCC was first found by Ohler et al. [11], from conserved upstream sequence blocks of orthologous, independently transcribed C. elegans and C. briggsae miRNA foldbacks and later, this same motif was found also by Zhou et al. [7]. Also, the motif TTTCAAAAA was found earlier by Ohler et al. [11] from the area $1000 \mathrm{bp}$ downstream of orthologous, independently transcribed C. elegans and C. briggsae miRNA foldbacks. We found this motif upstream in almost every C. elegans miRNA, including 16 miRNAs from the sense strand of an intron. This may not be surprising, because a motif very similar to this was discovered earlier from C. elegans protein coding gene introns [16]. Most miRNA genes have CT-repeats in their nearby upstream region, which may suggest significance of the motif GAAGGAGG in the studied area [7]. In contrast, the motif GANNNNGA has equal frequencies of different nucleotides in positions 3 to 6 , and any link to the CTrepeat is not, at this stage, obvious.

\section{Conservation studies of the motif GANNNNGA}

GANNNNGA was the only motif that occurred in all studied putative miRNA promoter sequences in both species. To determine whether this motif is specific to the studied area up to $1000 \mathrm{bp}$ upstream from the starting site of nematode miRNAs, we downloaded the sequences from 5000 upstream to 2000 bp downstream with respect to miRNA start sites for all miRNAs of both worms, and located all 
Table I: Significant patterns found with POCO from 1000 bp upstream sequences of $C$. elegans and $C$. briggsae

\begin{tabular}{|c|c|c|c|c|c|c|c|}
\hline Index & Pattern & C. elegans & C. briggsae & Index & Pattern & C. elegans & C. briggsae \\
\hline I & GAGA & $\mid 1.61$ & 15.38 & 34 & ANAGNNNC & 6.76 & 7.64 \\
\hline 2 & GANNNNGA & 11.29 & $|4.8|$ & 35 & GGNNGA & 6.63 & 8.29 \\
\hline 3 & GANANNG & 10.95 & 13.06 & 36 & GANGNG & 6.62 & 8.61 \\
\hline 4 & ANAGNG & 10.93 & 10.99 & 37 & GNANNNGG & 6.62 & 8.25 \\
\hline 5 & ANNNGNAG & 9.74 & 11.04 & 38 & GNANGNG & 6.27 & 7.42 \\
\hline 6 & GANNNAG & 9.70 & 11.64 & 39 & GNAGNNNG & 6.25 & 8.05 \\
\hline 7 & AGNGNNNA & 9.50 & 9.56 & 40 & GNNGNGNA & 5.99 & 8.04 \\
\hline 8 & ANGNNGA & 9.49 & 11.14 & 41 & AGNGNNC & 5.92 & 5.69 \\
\hline 9 & AGNGNA & 9.40 & 9.60 & 42 & GGNGG & 5.91 & 7.64 \\
\hline 10 & GAGNG & 9.36 & 9.48 & 43 & GNNANNGG & 5.91 & 6.96 \\
\hline II & AGNGA & 9.35 & 11.93 & 44 & ANNGGNNG & 5.79 & 7.29 \\
\hline 12 & ANNGNNGA & 9.01 & 10.00 & 45 & GNGNNGG & 5.73 & 7.65 \\
\hline 13 & GAANG & 8.85 & 11.34 & 46 & GNGNANG & 5.73 & 6.83 \\
\hline 14 & AGNAG & 8.75 & 11.58 & 47 & ANGNGG & 5.72 & 7.46 \\
\hline 15 & ANANGNG & 8.65 & 10.35 & 48 & ANNGGNG & 5.59 & 6.84 \\
\hline 16 & GNNNANGA & 8.53 & 11.19 & 49 & ANNGNGG & 5.56 & 6.56 \\
\hline 17 & AGNGNG & 8.44 & 8.23 & 50 & GGNNNANG & 5.48 & 7.39 \\
\hline 18 & ANNNAGNG & 8.33 & 10.28 & 51 & ANNNGGC & 5.45 & 5.79 \\
\hline 19 & GANGNNG & 8.17 & 10.05 & 52 & ANGNGNG & 5.36 & 7.00 \\
\hline 20 & GNGNGNG & 8.07 & 9.44 & 53 & AGGNG & 5.34 & 7.09 \\
\hline 21 & GNNGNNGA & 7.91 & 9.69 & 54 & GGNNNNGG & 5.28 & 7.55 \\
\hline 22 & ANGAG & 7.79 & 10.74 & 55 & GNGNNANG & 5.27 & 6.83 \\
\hline 23 & ANANGG & 7.78 & 9.83 & 56 & ANGGNG & 5.26 & 6.61 \\
\hline 24 & AAGNG & 7.75 & 10.14 & 57 & AGNGG & 5.21 & 6.80 \\
\hline 25 & GANNGNG & 7.65 & 8.82 & 58 & GGNNAG & 5.07 & 6.65 \\
\hline 26 & GNNNGAG & 7.59 & 9.32 & 59 & GTNGNNNG & 5.00 & 5.58 \\
\hline 27 & GNGAG & 7.58 & 9.06 & 60 & GGNNNNAG & 4.92 & 6.89 \\
\hline 28 & AGNNNGNG & 7.52 & 8.58 & 61 & ANGGNNNG & 4.89 & 6.69 \\
\hline 29 & GNNGAG & 7.41 & 9.65 & 62 & CGANNG & 4.86 & 5.27 \\
\hline 30 & AGNANNG & 7.41 & 9.22 & 63 & ANGNNNCG & 4.42 & 5.47 \\
\hline 31 & ANNGNGNG & 7.17 & 8.95 & 64 & GGNNNGG & 4.38 & 7.20 \\
\hline 32 & GNNGNGA & 6.92 & 8.44 & 65 & ANNNGGNC & 4.26 & 5.18 \\
\hline 33 & AGNNNAG & 6.80 & 8.61 & & & & \\
\hline
\end{tabular}

The patterns are listed in the order of their average occurrences.

the motif occurrences from this area. Further, we drew the 200 bp moving average curves of the frequency of motif GANNNNGA for both worms in this larger area around the miRNAs (Figure 3 ). The curves show that the motif GANNNNGA has conserved distribution relative to miRNA starting sites in both worms, and that it is most frequent in the area 1000 bp upstream from the miRNA start site with a pronounced peak at about $500 \mathrm{bp}$ with $\sim 13$ occurrences on average in C. elegans and 15 occurrences in C. briggsae, while the overall average count of the motif is less than 10 in both worms.

All occurrences of the motif GANNNNGA were then counted from 1000 bp upstream of 70 orthologous miRNA pairs in C. elegans and C. briggsae. The results of this calculation and the location of each miRNA with respect to the C. elegans protein coding genes are presented in Table 2. For those miRNAs that are from the protein coding area, we also indicate its strand with respect to the host gene (sense +, antisense -). Fifteen orthologous
miRNA pairs have 15 or more instances of the motif GANNNNGA in the area 1000 bp upstream from their start sites, including 7 miRNAs from the sense strand of an intron and 1 miRNA overlapping an exonic area. On the other hand, 34 of the 70 orthologous miRNAs have less than 10 motif GANNNNGA occurrences in C. elegans. When comparing these results, we found that the motif GANNNNGA is especially abundant in the $1000 \mathrm{bp}$ upstream sequences of miRNAs mir-1, mir-124 and mir228 in both worms. From these, mir-1 and mir-228 are intergenic, while mir-124 is located in the intron of the gene trpa-1.

To determine whether the motif GANNNGA is conserved in the upstream sequences of mir-1, mir-124 and mir-228 orthologs in other species, we located all its occurrences from 1000 bp upstream sequences of all miRNAs that, according to miRBase [17], belong to mir-1 or mir-124 family in human and mouse genomes. Mir-228 is not found from these two genomes, and generally it is 


\begin{tabular}{|c|c|c|c|c|c|}
\hline Tool & Consensus & $\log 0$ & \#cel (100) & \#cbr (95) & p-value \\
\hline POCO & GANNNNGA & & $1129 / 100$ & $1461 / 95$ & 9.3E-09 \\
\hline Weeder & GAAGGAGG & & $473 / 94$ & $623 / 93$ & $3.3 \mathrm{E}-05$ \\
\hline $\begin{array}{l}\text { Gibbs } \\
\text { Motif } \\
\text { Sampler }\end{array}$ & TTTCAAAAA & & $1218 / 98$ & $875 / 94$ & $1.8 \mathrm{E}-10$ \\
\hline MEME & CTCCGCCC & & $153 / 57$ & $220 / 71$ & $5.0 \mathrm{E}-08$ \\
\hline & TTTCAAAA & & $108 / 63$ & $86 / 45$ & 2.4E-11 \\
\hline
\end{tabular}

\section{Figure 2}

Significant conserved motifs discovered from the miRNA regulatory region of $C$. elegans and $C$. briggsae. Presented is the total number of the motif instances in both regulatory region sequence sets and the number of miRNAs that contain the motif in their upstream region. The rightmost column shows the $p$-value of the motif calculated against random background sequences. Motif logos show the information content present at every position in the sequence, for example in the logo of motif GANNNNGA the G:s and A:s are certain, thus having the highest information content ( 2 bits) while in the positions 3 to 6 all four nucleotides have about the same frequencies and the information content in these positions is close to zero.

included in the mir-124 family [18]. Both human and mouse, have three miRNAs belonging to the mir-1 -family: mir-1-1, mir-1-2 and mir-206. The 1000 bp upstream sequence of human hsa-mir-1-1 includes 21 occurrences of the motif which is more than twice as many occurrences as the corresponding sequences of hsa-mir-1-2 and hsamir-206 which contain 10 and 6 occurrences, respectively. For mouse, the numbers are 20 for mmu-mir-1-1, 6 for mmu-mir-1-2, and 6 for mmu-mir-206. Similar results were observed when looking at the occurrences of GANNNNGA in the mir-124 -family, where the putative promoter sequence of mir-124a-1 is the only one that includes a significant number of the motif in both species, 37 and 19, respectively. In summary, the mir-1-1 1000 bp upstream sequences of human and mouse contain nearly as many occurrences of the motif GANNNNGA (21 and $20)$ as the corresponding sequences of mir-1 of C. elegans and C. briggsae (23 and 26). The same holds for the 1000 bp upstream sequences of mir-124-a1 of human and mouse (37 and 19) compared with the corresponding sequences for mir-124 of C. elegans and C. briggsae (26 and 30 ). On average, the human miRNA 1000 bp upstream sequences contain 9.9 occurrences of motif GANNNNGA and in mouse the corresponding average is 10.6 compared to 11.3 and 14.8 for C. elegans and C. briggsae, respectively.

We draw the frequency diagrams of the motif GANNNNGA in the $1000 \mathrm{bp}$ upstream sequences of mir1 and mir-124 orthologs in these four species (Figure 4), and made the global alignments of mir-1 and mir-124 upstream sequences (Additional files 1 and 2). In both cases, we chose mir-1-1 as the representative of mir-1 -family and mir-124a-1 for the representative of mir-124 -family for human and mouse. The distribution of motif GANNNNGA in the 1000 bp upstream area of both miRNAs looks rather similar, giving a clue to possible conser- 
(a)

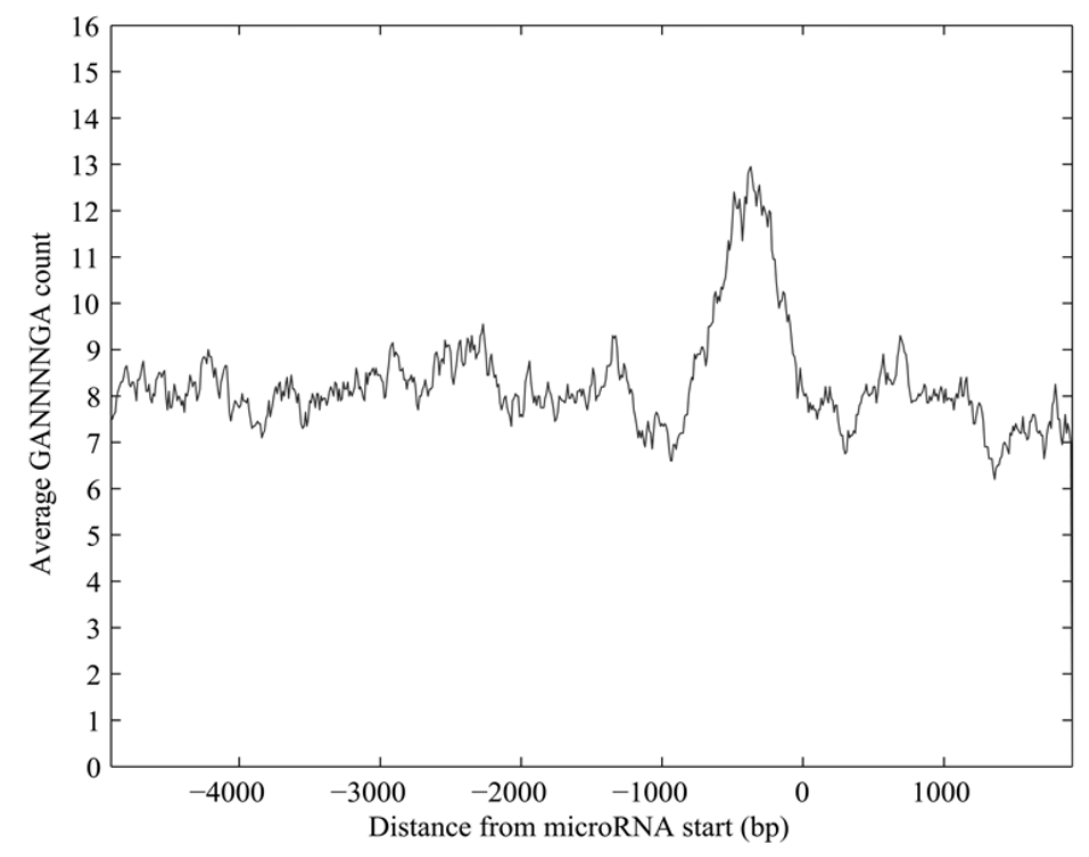

(b)

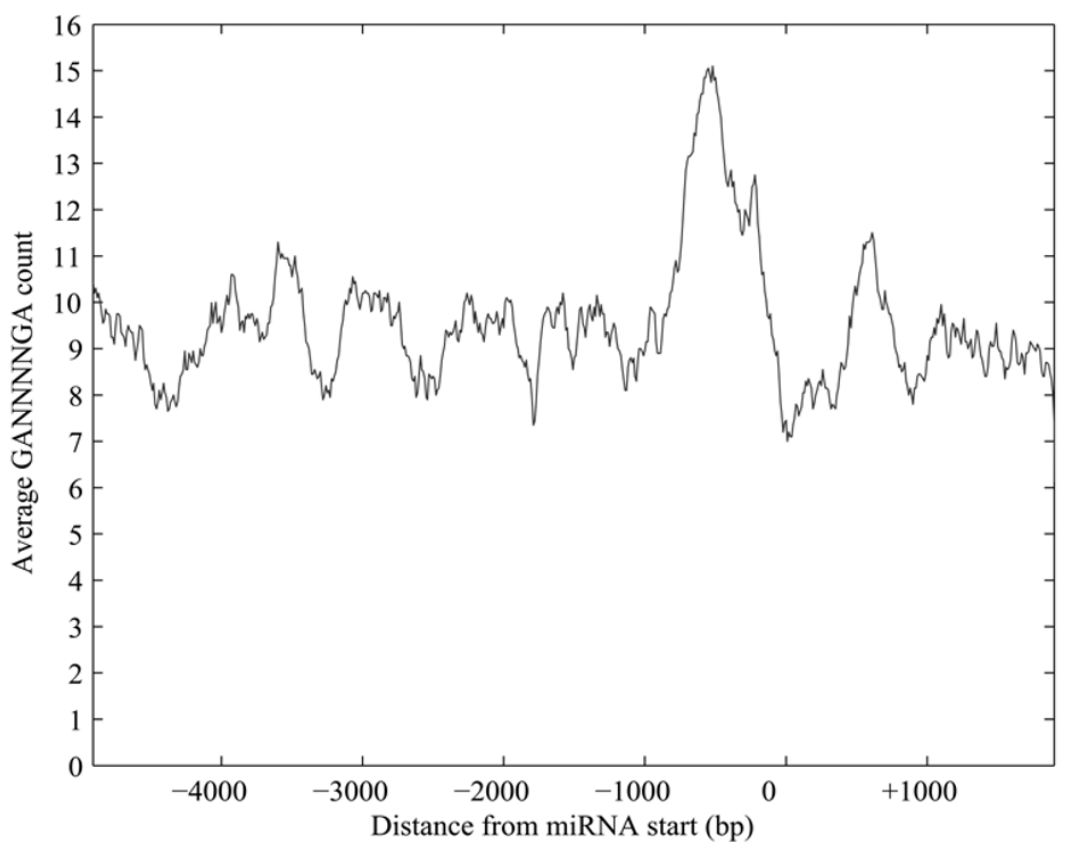

Figure 3

The frequency distribution of motif GANNNNGA around the miRNAs of $C$. elegans (a) and $C$. briggsae (b). All occurrences of the motif GANNNNGA were located from the sequences 0-5000 bp upstream and 0-2000 bp downstream of all $C$. elegans and $C$. briggsae miRNA start sites and the corresponding 200 bp central moving average curves were drawn for both worms. 
Table 2: Motif count of GANNNNGA in the 1000 bp upstream sequences of orthologous miRNAs of $C$. elegans and $C$. briggsae

\begin{tabular}{|c|c|c|c|c|c|c|c|c|c|}
\hline Index & miRNA & CEL gene relationship & CEL & CBR & Index & miRNA & CEL gene relationship & CEL & CBR \\
\hline I & mir-228 & intergenic & 52 & 29 & 36 & mir-268 & Intergenic & 10 & 23 \\
\hline 2 & $\operatorname{mir}-47$ & Intergenic & 27 & 29 & 37 & mir-70 & Intronic + & 9 & 9 \\
\hline 3 & mir-233 & Intronic + & 26 & 16 & 38 & mir-253 & Intronic + & 9 & 10 \\
\hline 4 & mir-124 & Intronic + & 26 & 30 & 39 & mir-353 & Intronic + & 9 & 10 \\
\hline 5 & mir-239.2 & Intergenic & 24 & 13 & 40 & mir-359 & Intergenic & 9 & 10 \\
\hline 6 & mir-24I & Intergenic & 23 & 10 & 41 & mir-42 & Intergenic & 9 & 19 \\
\hline 7 & mir-52 & Intergenic & 23 & 25 & 42 & mir-254 & Intronic + & 8 & 6 \\
\hline 8 & mir-I & Intergenic & 23 & 26 & 43 & mir-240 & Intergenic & 8 & 7 \\
\hline 9 & mir-239.I & Intergenic & 22 & 13 & 44 & mir-45 & Intergenic & 8 & 10 \\
\hline 10 & mir-87 & Intronic + & 18 & 15 & 45 & mir-57 & Intergenic & 8 & 18 \\
\hline II & mir-62 & exon overlap & 18 & 21 & 46 & mir-79 & Intergenic & 8 & 34 \\
\hline 12 & mir-7I & Intronic + & 18 & 34 & 47 & mir-360 & Intergenic & 7 & 8 \\
\hline 13 & mir-58 & Intronic + & 17 & 16 & 48 & mir-235 & Intergenic & 7 & 9 \\
\hline 14 & mir-82 & Intronic + & 17 & 22 & 49 & mir-248 & Intergenic & 7 & 10 \\
\hline 15 & lin-4 & Intergenic & 17 & 23 & 50 & mir-232 & Intergenic & 7 & II \\
\hline 16 & mir-86 & Intronic - & 17 & 24 & 51 & mir-34 & Intergenic & 7 & 12 \\
\hline 17 & mir-49 & Intergenic & 16 & 10 & 52 & mir-242 & Intergenic & 7 & 14 \\
\hline 18 & mir-85 & intronic + & 16 & 13 & 53 & mir-8I & Intronic - & 7 & 14 \\
\hline 19 & mir-354 & 3' UTR + & 16 & 14 & 54 & mir-245 & Intergenic & 7 & 15 \\
\hline 20 & mir-90 & Intergenic & 16 & 27 & 55 & mir-23I & Intergenic & 6 & 10 \\
\hline 21 & mir-77 & Intergenic & 15 & 8 & 56 & mir-60 & Intergenic & 6 & 12 \\
\hline 22 & mir-35 & intronic - & 15 & 11 & 57 & mir-5I & Intergenic & 6 & 18 \\
\hline 23 & mir-244 & Intergenic & 15 & 16 & 58 & mir-83 & Intergenic & 6 & 18 \\
\hline 24 & mir-84 & Intergenic & 14 & 18 & 59 & mir-6I & Intergenic & 6 & 21 \\
\hline 25 & mir-25I & Intergenic & 13 & 9 & 60 & mir-392 & Intergenic & 5 & 5 \\
\hline 26 & mir-80 & Intergenic & 13 & 15 & 61 & mir-234 & Intergenic & 5 & 9 \\
\hline 27 & mir-73 & Intergenic & 13 & 16 & 62 & mir-356 & 3' UTR + & 5 & 9 \\
\hline 28 & mir-50 & Intronic + & 12 & 11 & 63 & mir-355 & Intergenic & 5 & 10 \\
\hline 29 & mir-259 & Intergenic & 12 & 13 & 64 & mir-358 & Intronic - & 5 & 10 \\
\hline 30 & $\operatorname{mir}-67$ & Intronic + & 12 & 18 & 65 & mir-236 & Intergenic & 5 & 11 \\
\hline 31 & mir-46 & Intergenic & 11 & 19 & 66 & mir-72 & Intergenic & 5 & 14 \\
\hline 32 & let-7 & Intergenic & 10 & 5 & 67 & mir-230 & Intergenic & 5 & 15 \\
\hline 33 & mir-255 & Intergenic & 10 & 9 & 68 & Isy-6 & Intergenic & 5 & 18 \\
\hline 34 & mir-246 & Intergenic & 10 & 10 & 69 & mir-75 & Intergenic & 4 & 14 \\
\hline 35 & mir-252 & Intergenic & 10 & 16 & 70 & mir-249 & Intergenic & 3 & 12 \\
\hline
\end{tabular}

Included in the table is the location of each miRNA with respect to $C$. elegans protein coding genes and the strand of the miRNAs in the protein coding area relative to their host gene. CEL gene relationship indicates the genomic location. "+" or "-" indicates the strand location. CEL, $C$. elegans; CBR, C. briggsae.

vation of the motif even between these phylogenetically distant species.

Using miRNA microarray analysis, we measured the signal intensity of C. elegans miRNAs isolated from age synchronized whole body wildtype worms and plotted the values versus the number of observed GANNNNGA motifs for each miRNA (Figure 5). A weak correlation only was found, $\mathrm{r} 2=0.13$.

\section{Discussion}

In this study, we examined the $1000 \mathrm{bp}$ upstream regions of all C. elegans and C. briggsae miRNAs to reveal characteristic sequence motifs. In addition to the miRNAs in the intergenic area, we included in our study also the miRNAs that lie in the protein coding area and whose possible pro- moters were not studied earlier. In the search, we focused on over-represented, phylogenetically conserved short sequence motifs that can be found from the miRNA upstream regions, independently of miRNA origins in the genome. The four motif finding algorithms used in the search gave a couple of very frequent result patterns whose consensus sequences were quite diverse, due to the different motif and background models used in the algorithms.

We found one significant sequence motif, GANNNNGA, which was quite abundant and could be located from all miRNA upstream sequences of C. elegans and C. briggsae. Thus, the motif GANNNNGA is found from all studied miRNA upstream regions, and no clear difference can be made in its abundance when comparing the intergenic miRNAs and the miRNAs located in the protein coding 
(a)

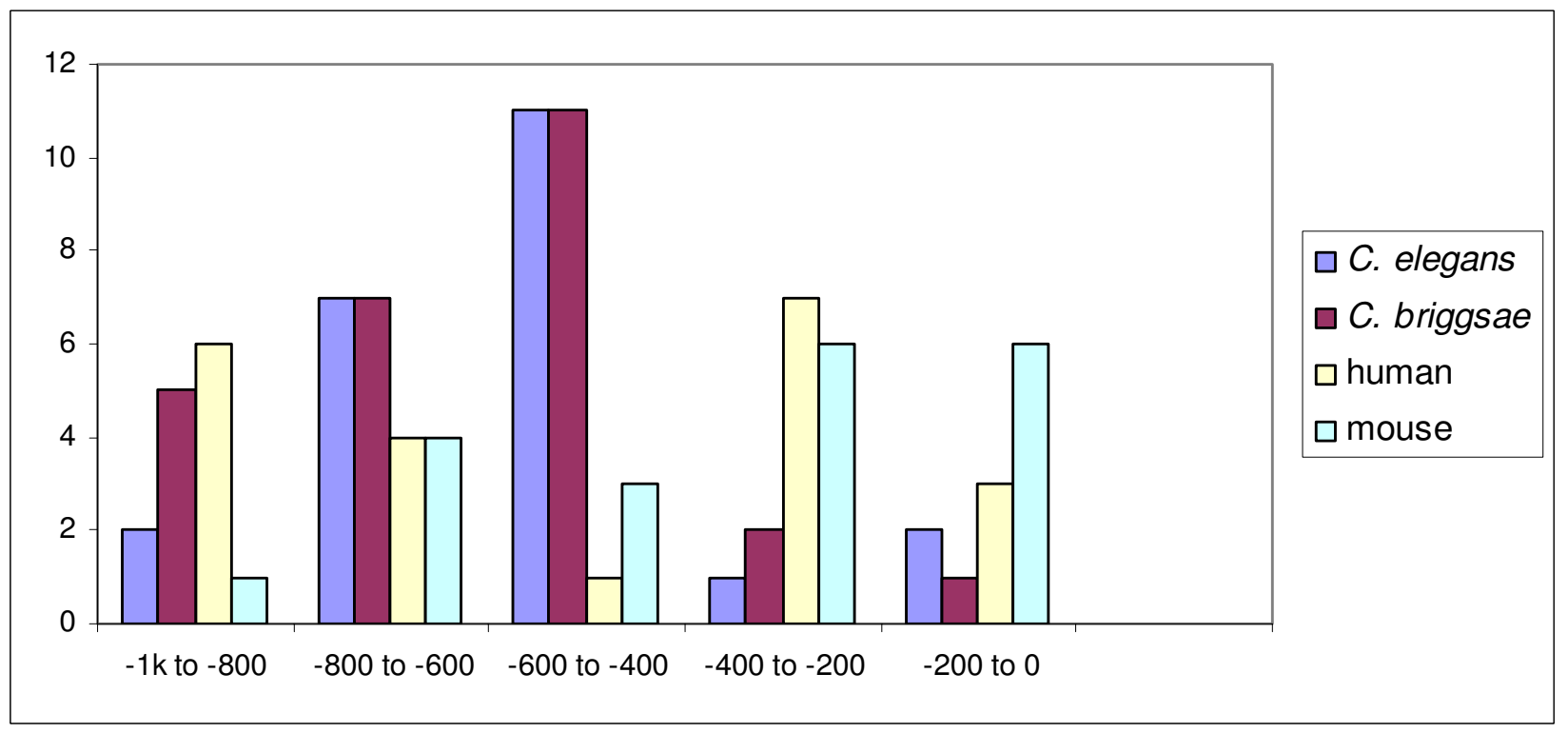

(b)

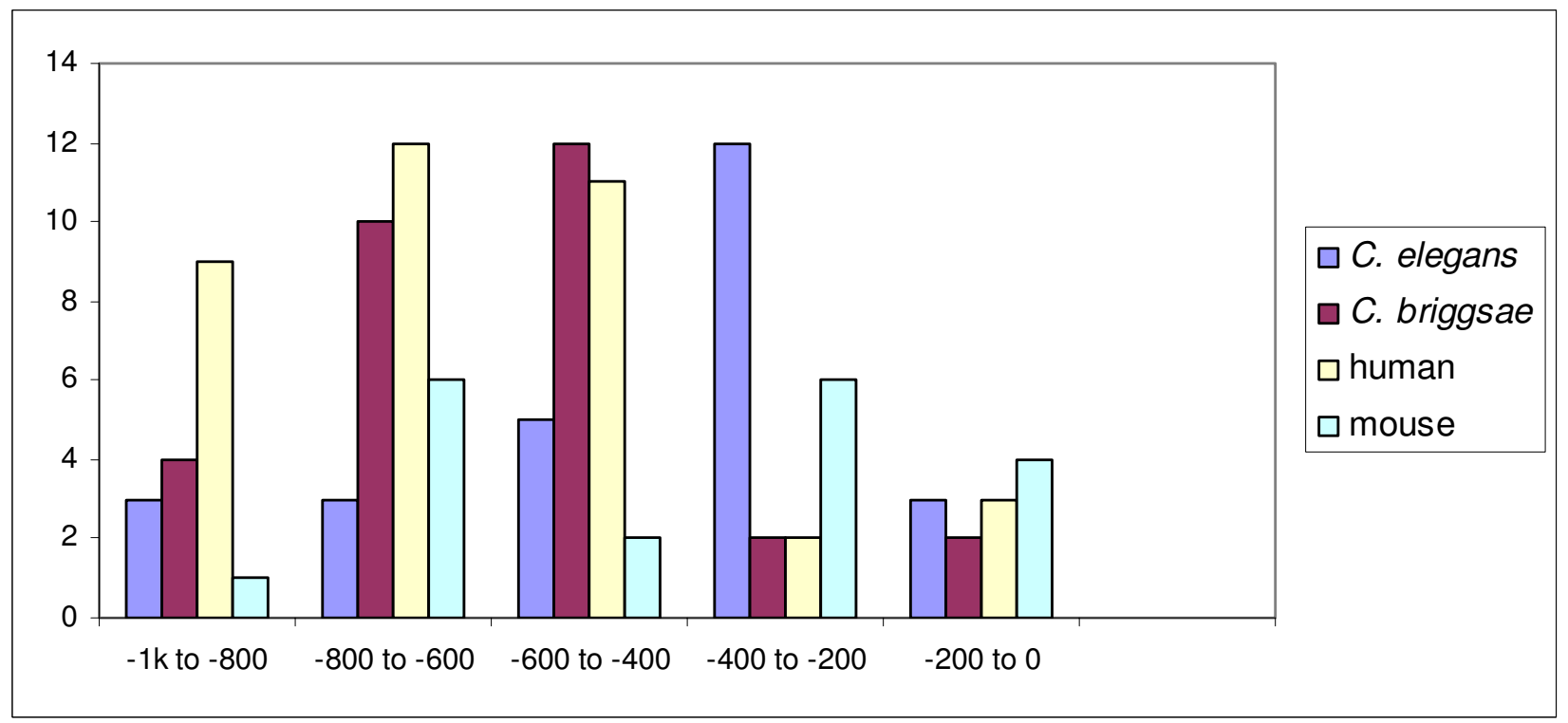

Figure 4

The frequency diagrams of the motif GANNNNGA occurrences in sequences 1000 bp upstream of mir-I (panel a) and mir-I24 (panel b) family members in C. elegans, $C$. briggsae, human and mouse. The mir- $I-I$ and mirI24-al 1000 bp upstream sequences of human and mouse contain similar number of occurrences of the motif GANNNNGA as the corresponding sequences in C. elegans and C. briggsae. 


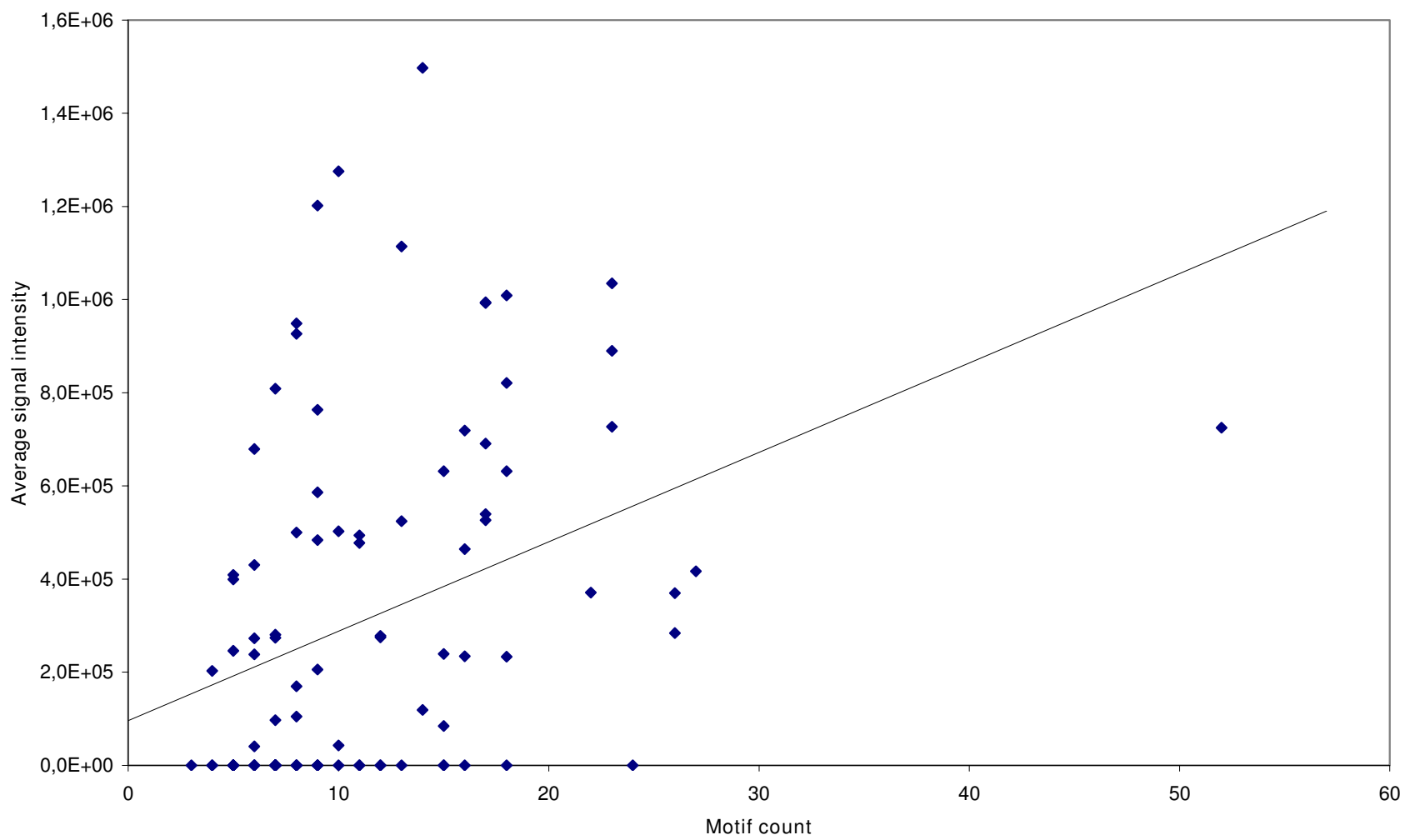

Figure 5

miRNA microarray expression analysis in C. elegans. Dots represent the absolute expression level (vertical axis) of wildtype N2 C. elegans miRNAs from whole L4 stage animals as a function of the number of GANNNNGA motifs (horizontal axis). The line indicates a regression plot of the data $\left(r^{2}=0.13\right)$.

area. In addition, this pattern seemed to also have a conserved distribution in the area surrounding the studied $1000 \mathrm{bp}$ miRNA upstream loci in these two worms, having most of its occurrences between $0-1000$ bp upstream from miRNA start sites. From earlier studies [11], this is known to be a highly conserved region upstream of intergenic C. elegans and C. briggsae orthologous miRNA precursors, and the putative PolII type promoter sequences of most C. elegans intergenic miRNAs are predicted to be situated on the $0-500 \mathrm{bp}$ area upstream from the precursors [7]. The motif GANNNNGA was not found in earlier studies of $C$. elegans miRNA promoters $[7,11]$ perhaps because these studies were focused on the intergenic miRNA promoters, thus leaving motifs in the coding area out of scope. Another reason is the tools used for motif finding had a motif model different from that used in POCO, which puts the wildcards $(\mathrm{N})$ in certain places in the middle of the motif and keeps the other places occupied with constant nucleotides [19].

Several lines of evidence suggest that the motif GANNNNGA is not a miRNA promoter. First, although the motif is found proximal to the miRNA transcript, it is found in abundant copies, which is more characteristic of enhancer or other regulatory elements. Second, we have cloned the $1000 \mathrm{bp} 5$ ' flanking region of mir-1 and used it to construct an expression plasmid with green fluorescent protein as the reporter (data not shown). We were not able to express the reporter despite successful experiments with a variety of other promoters [20]. Eventually, large scale deletion analysis of multiple miRNA flanking sequences using a strong promoter and including the miRNA sequence may be necessary to help define the context where GANNNNGA is active. Thirdly, there appears to be only a weak correlation between the number of GANNNNGA motifs and absolute expression levels (Figure 5). Finally, using STAMP [21], we were not able to find any matching transcription factor binding sites in C. elegans using the TRANSFAC or JASPAR databases.

Prior to and independent from the current study, there has been keen interest in mir-1 and mir-124, two miRNAs with the most abundant GANNNNGA motifs. These two miRNAs are found from all bilateria and are thus interpreted to be among those most phylogenetically ancient miRNAs $[22,23]$. Both are known to have high tissue spe- 
cificity. Mir-1 is known to have high expression levels in mammalian heart and muscle cells while mir-124 contributes to the cells of the nervous system [24]. In a landmark study, Lim et al. [5] demonstrated through transfection to human HeLa cells, that these miRNAs can downregulate the transcription of 96 and 174 annotated genes, respectively, most likely through 3' UTR seed matches, and thus may have a wide global effect to the identity of the tissues mentioned above [5]. The down regulation of brain mRNAs by mir-124 in transfected HeLa cells suggests that this miRNA confers tissue specific expression. Consistent with this result, the transfection of mir-1 duplexes to HeLa cells produced a heart and skeletal muscle profile. Mir-124 has also been shown to promote neuronal differentiation by down regulating the general splice regulator PTB1 [25]. From the evolutionary point of view, the tight tissue specific regulation and large number of genes targeted appears logical, since phylogenetically ancient miRNAs are supposed to have more targets than more recent ones [22]. Consistent with these studies, we found that another brain specific miRNA, hsa-mir-9, has 21 occurrences of motif GANNNNGA in its 1000 bp upstream sequence (data not shown). Thus, the presence of more copies of GANNNNGA in the upstream sequences of miRNAs may signal a globally conserved function, such as tissue specific expression. We also found the frequency distribution of the motif in the upstream sequences from human and mouse orthologs of mir-1 and mir-124 to approximately follow its corresponding distributions in the two worms, thus confirming the conserved nature of the motif.

Motifs CTCTCTCTCTC and CTTCTTCTTCTTC, complementary to the motif GANNNNGA were found earlier in the studies of $C$. elegans muscle specific regulatory motifs [26]. We found these motifs also from the mir-1 and mir1241000 bp upstream sequences in C. elegans and $C$. briggsae, thus strengthening the connection of these miRNAs with a muscle specific function in these two worms.

Computational identification of miRNA genes relies upon the properties of known miRNAs such as the length of mature miRNA and their hairpin precursor secondary structure, together with high phylogenetic conservation of some miRNAs $[18,27,28]$. The predictions based on the miRNA hairpin sequence only are not very accurate and tend to give a high number of false positives. Computational algorithms are also prone to false negatives and may miss miRNAs that do not have clear homologs in related species. The accuracy of the algorithms can be improved by adding characteristics from miRNA flanking sequence, such as scoring the sequence conservation and characteristic motifs [11]. Ohler and his co-workers successfully applied knowledge about the motif CTCCGCCC and its location upstream from the orthologous inde- pendently transcribed miRNA foldbacks to improve the accuracy of the miRNA gene prediction. In our study, this motif was found also from upstream of 10 C. elegans miRNAs located in the sense strand of an intron (data not shown), thus suggesting that it could be used also to improve prediction of the intronic miRNAs. In addition, the motif GANNNNGA is highly conserved in the vicinity of the miRNA starting site and equally frequent in the upstream of all the miRNAs despite their genomic location, suggesting that it could be a useful additional characteristic for the search and computational prediction of miRNA sequences.

\section{Conclusion}

To better understand the transcriptional regulation of miRNAs, we searched for conserved sequence motifs from the area 1000 bp upstream from all miRNAs independent of their genomic location. We discovered one significant sequence motif GANNNNGA, occurring in every miRNA upstream sequence and having a conserved distribution in the upstream sequences of all miRNAs of nematode worms C. elegans and C. briggsae. It appears to be a common factor for the upstream regions of all miRNAs and is concentrated in a proximal area near the beginning of the miRNA precursor start sites. It may not be a transcription factor binding site, but rather a co-factor site, or recognition sequence for miRNA processing. The abundance and conservation of this motif in the upstream of two old and important miRNAs, mir-1 and mir-124 suggest a connection to miRNAs with global specialized function. The abundance and location of the GANNNNGA motif may be of benefit in computational prediction of miRNAs.

\section{Methods \\ Datasets}

We downloaded from WormBase release WS160, 31 July 2006 [29] the sequences $1000 \mathrm{bp}$ upstream from the beginning of all available C. elegans and C. briggsae miRNAs. We considered as clustered those C. elegans miRNAs that were less than $1000 \mathrm{bp}$ distance from each other. Seven clusters containing 23 miRNAs in total were found and only the upstream sequence of one, upstream-most miRNA from each cluster, was chosen to represent the whole cluster. Thus, the number of C. elegans $1000 \mathrm{bp}$ miRNA upstream sequences was reduced from 116 to 100 while for C. briggsae, there were 95 miRNAs in all. In this study, these two datasets were considered as putative miRNA regulatory sequence sets. From the 100 C. elegans miRNAs included into this study, 75 are located into the noncoding area of the genome and 25 are located in the gene coding area. We selected the $1000 \mathrm{bp}$ upstream from intergenic miRNAs, rather than from their host genes, in order to find regulatory motifs specific for miRNA sequences. 


\section{Motif extraction}

Within both of our data sets we searched for short, overrepresented patterns to find putative regulation motifs. For this purpose we used four motif finding tools available: POCO, Weeder, MEME and Gibbs Motif Sampler. POCO [19], takes advantage of exhaustive search methods and bootstrapping to find significant patterns composed of the nucleotides $\mathrm{A}, \mathrm{C}, \mathrm{G}, \mathrm{T}$ and $\mathrm{N}$ from a given data set compared with the selected background organism. The resulting patterns contain at least 4 known nucleotides in steady places while the first or the last nucleotide must not be the wildcard N. Thus, mismatches are allowed only in fixed places in the pattern. All the full-length patterns and their sub-patterns are automatically analyzed, so when the maximum possible length of searched patterns, $8 \mathrm{bp}$, is selected for pattern length, the output contains all significant patterns which are 4-8 bp long. We used POCO in our miRNA upstream sequence motif analysis because this kind of motif model has not been used earlier in similar studies $[7,11-13]$, and so it could give a new perspective to the possible regulative elements of miRNA genes. For the background organism to be used in the analysis we chose C. elegans clean which contains the promoter regions (downloaded from Ensmart [30], parameters: known genes, one output per gene, 5 ' upstream only and 5' Flank 3000 bp [19]) of almost every gene in the C. elegans genome where sequences containing letters other than A, C, G or T are removed. We chose the maximum 8 for pattern length and left all the remaining parameters as default. Weeder [31,32] is an exhaustive enumeration method for finding approximate occurrences of patterns. Significance of the found motif is calculated against the oligo frequency in the protein coding gene upstream regions of the selected organism. We chose to use Weeder in this study, because it has proved to perform well when compared to other common motif finding tools [33]. We ran Weeder with "quick" mode, and looked for motifs of length 8 allowing 2 mutations. MEME [34] is a probabilistic local alignment based method for discovering degenerate sequence motifs. The significance of the found motif is calculated against random sequences with the same nucleotide composition as the query sequences, so the motifs E-value refer to the expected number of motifs of equal width with the same or higher likelihood in a random sequence set with the same size and nucleotide composition as the input sequence set. We used MEME because it is used also in the other miRNA upstream motif studies $[7,11,13]$, which gives a possibility to compare the results. In this study, MEME was launched to search motifs with width 8 bp having any number of repetitions in the input sequence set. The optional attributes were left as default. The Gibbs Motif Sampler is a probabilistic, alignment based approach for locating common motifs in collections of sequences [35]. The Gibbs Recursive Sampler [36] is a version of Gibbs sampler, developed for locating multiple transcription factor binding sites simultaneously from unaligned heterogenous DNA sequences. In this study, we launched the Gibbs Recursive Sampler with the minimum attributes: the number of different motifs was set to 3 and for each of them the initial width estimate was set to 8 , and the maximum number of sites per sequence was set to 10 . Even though the initial width of the sites was entered as 8 , the output sites had length of 8 or more, where 8 columns were conserved and the rest of them were non-conserved.

\section{Motif analysis}

From POCO results for both worms, we chose such patterns that were found from every sequence in the putative miRNA regulatory sequence sets, having $p$-values less than 0.05 and searched among them for patterns common to both sets (for algorithm, see Additional file 3. These patterns were then aligned with ClustalW [37], (Additional file 4).

The most significant motifs were located from the putative miRNA regulatory region with Visualize tool of POXO tool series [38], or Weeder Motif Locator included in MoD Tools [32], allowing two mismatches to the consensus sequence, and the position weight matrices were calculated from these results. The motif logos were created with WebLogo [39] and Transfac and Jaspar databases were searched for matching TFBSs with STAMP [21].

To test the statistical significances of the found motifs, we generated 100 random 1000 bp long sequences with the same nucleotide composition as C. elegans genome and counted the motif occurrences from these sequences. Then we calculated the $p$-values for the putative motifs with the independent samples t-test.

The 1000 bp upstream sequences of human and mouse miRNAs that belong to mir-1 and mir-124 families were downloaded from UCSC Genome Browser [40], and the multiple sequence alignments for the mir-1 and mir-124 families upstream sequences were made with ClustalW [37].

Additional file 5 shows the detailed workflow of the discovery and study of motif GANNNNGA.

\section{miRNA microarray analysis}

Wild type worms (strain N2) were fed with OP50 E. coli on culturing plates containing Nematode Growth Media (NGM) agar [41]. Animals were grown at $20^{\circ} \mathrm{C}$, synchronized and harvested at Larva 4 (L4) stage. mirVanaTM miRNA Isolation-protocol (Ambion Inc., Austin, Texas) was used to isolate small ( $<200$ nucleotide) RNAs containing miRNAs which were labeled using NCodeTM miRNA Labeling System (Invitrogen, Carlsbad, CA) using 
the manufacturer's protocol. Labelled RNA was hybridized on NCodeTM Multi-Species miRNA Microarray V2arrays (Invitrogen) containing three sub arrays (technical replicates) with 115 probes for C. elegans miRNAs. Arrays were scanned using ScanArray 5000 (GSI Lumonics, Billerica, MA) and images were converted as numerical form using TIGR Spotfinder- software [42] resulting in raw signal intensity values. Mean signal value for each miRNA was calculated from seven independently hybridized arrays.

\section{Authors' contributions}

LH planned and conducted the experiments, and wrote the manuscript. SA conducted experiments and contributed to writing the manuscript. GW conceived the study, planned the experiments, and wrote the manuscript.

\section{Additional material}

\section{Additional file 1}

The multiple sequence alignment of the mir-1 family upstream sequences of $\mathrm{C}$. elegans, C. briggsae, human and mouse. Click here for file

[http://www.biomedcentral.com/content/supplementary/14712199-9-105-S1.doc]

\section{Additional file 2}

The multiple sequence alignment of the mir-124 family upstream sequences of $\mathrm{C}$. elegans, C. briggsae, human and mouse.

Click here for file

[http://www.biomedcentral.com/content/supplementary/14712199-9-105-S2.doc]

\section{Additional file 3}

The algorithm applied to find common patterns from two sets. Click here for file

[http://www.biomedcentral.com/content/supplementary/14712199-9-105-S3.doc]

\section{Additional file 4}

The multiple sequence alignment of conserved patterns found with POCO.

Click here for file

[http://www.biomedcentral.com/content/supplementary/1471-

2199-9-105-S4.doc]

\section{Additional file 5}

The detailed workflow of the discovery and study of motif

GANNNNGA.

Click here for file

[http://www.biomedcentral.com/content/supplementary/14712199-9-105-S5.doc]

\section{Acknowledgements}

The authors wish to thank Matti Kankainen, Vuokko Aarnio, and Merja Lakso for help and critical reading of the manuscript. This study was supported by the Saastamoinen Foundation, the North Savo Regional Fund of the Finnish Cultural Foundation, and the Finnish Graduate School of Neuroscience.

\section{References}

I. Lee Y, Kim M, Han J, Yeom K, Lee S, Baek S, Kim V: MicroRNA genes are transcribed by RNA polymerase II. EMBO J 2004, 23:405I-4060.

2. Bartel DP: MicroRNAs: genomics, biogenesis, mechanism and function. Cell 2004, I I 6:28I-297.

3. Kong $Y$, Han J: MicroRNA: Biological and computational perspective. Genomics Proteomics Bioinformatics 2005, 3:62-72

4. Lewis B, Shih I, Jones-Rhoades M, Bartel D, Burge C: Prediction of mammalian microRNA targets. Cell 2003, I I 5:787-798.

5. Lim L, Lau N, Garrett-Engele P, Grimson A, Schelter J, Castle J, Bartel $D P$, Linsley $P$, Johnson J: Microarray analysis shows that some microRNAs downregulate large numbers of target mRNAs. Nature 2005, 433:769-773.

6. Lee Y, Kim M, Han J, Yeom KH, Lee S, Baek S, Kim V: MicroRNA genes are transcribed by RNA polymerase II. EMBO J 2004, 23:405I-60.

7. Zhou X, Ruan J, Wang G, Zhang W: Characterization and identification of microRNA core promoters in four model species. PLoS Computational Biology 2007, 3:04 I2-0423.

8. Borchert $G$, Lanier W, Davidson B: RNA polymerase III transcribes human microRNAs. Nat Struct Mol Biol 2006, 13:1097-II0I.

9. Lagos-Quintana M, Rauhut R, Lendeckel W, Tuschl T: Identification of novel genes coding for small expressed RNAs. Science 200I, 294:853-858.

10. Lau N, Lim L, Weinstein E, Bartel DP: An abundant class of tiny RNAs with probable regulatory roles in Caenorhabditis elegans. Science 200I, 294:858-862.

II. Ohler U, Yekta S, Lim L, Bartel DP, Burge C: Patterns of flanking sequence conservation and a characteristic upstream motif for MicroRNA gene identification. RNA 2004, 10:1309-1 322.

12. Megraw M, Baev V, Rusinov V, Jensen S, Kalantidis K, Hatzigeorgiou A: MicroRNA promoter element discovery in Arabidopsis. RNA 2006, 12:1612-1619.

13. Sethupathy P, Megraw M, Barrasa M, Hatzigeorgiou A: Computational Identification of Regulatory Factors Involved in MicroRNA Transcription. LNCS 2005, 3746:457-468.

14. Matys V, Kel-Margoulis O, Fricke E, Liebich I, Land S, Barre-Dirrie A, Reuter I, Chekmenev D, Krull M, Hornischer K, Voss N, Stegmaier P, Lewicki-Potapov B, Saxel H, Kel A, Wingender E: TRANSFAC and its module TRANSCompel: transcriptional gene regulation in eukaryotes. Nucleic Acids Res 2006, 34:D 108-1 IO.

15. Vlieghe D, Sandelin A, De Bleser PJ, Vleminckx K, Wasserman WW, van Roy F, Lenhard B: A new generation of JASPAR, the openaccess repository for transcription factor binding site profiles. Nucleic Acids Res 2006:D95-7.

16. Lim $L$, Burge $C$ : A computational analysis of sequence features involved in recognition of short introns. Proc Natl Acad Sci USA 2001, 98: III93-III98.

17. Griffiths-Jones S, Grocock R, van Dongen S, Bateman A, Enright A: miRBase: microRNA sequences, targets and gene nomenclature. Nucleic Acids Res 2006, 34:D I40-D I 44.

18. Lim L, Lau N, Weinstein E, Abdelhakim A, Yekta S, Rhoades M, Burge C, Bartel DP: The microRNAs of Caenorhabditis elegans. Genes Dev 2003, 17:991-1008.

19. Kankainen M, Holm L: POCO: discovery of regulatory patterns from promoters of oppositely expressed gene sets. Nucleic Acids Res 2005:W427-43I.

20. Lakso M, Vartiainen S, Moilanen A-M, Sirviö J, Thomas JH, Nass R, Blakely RD, Wong G: Dopaminergic neuronal loss and motor deficits in Caenorhabditis elegans overexpressing human alpha-synuclein. J Neurochem 2003, 86:165-172.

21. Mahony S, Auron P, Benos P: DNA Familial Binding Profiles Made Easy: Comparison of Various Motif Alignment and Clustering Strategies. PLoS Comput Biol 2007, 3:0578-059I.

22. Sempere L, Cole C, McPeek M, Peterson K: The Phylogenetic Distribution of Metazoan microRNAs: Insights into Evolutionary Complexity and Constraint. J Exp Zoolog B Mol Dev Evol 2006, 306B:575-588.

23. Prochnik S, Rokhsar D, Aboobaker A: Evidence for a microRNA expansion in the bilaterian ancestor. Dev Genes Evol 2007, 217:73-77. 
24. Lagos-Quintana M, Rauhut R, Yalcin A, Meyer J, Lendeckel W, Tuschl $\mathrm{T}$ : Identification of Tissue-Specific MicroRNAs from Mouse. Current Biology 2002, I 2:735-739.

25. Makeyev EV, Zhang J, Carrasco MA, Maniatis T: The MicroRNA miR-I24 promotes neuronal differentiation by triggering brain-specific alternative pre-mRNA splicing. Mol Cell 2007, 27:435-48.

26. Zhao G, Schriefer L, Stormo G: Identification of muscle-specific regulatory modules in Caenorhabditis elegans. Genome Res 2007, I 7:348-57.

27. Ambros V, Lee R, Lavanway A, Williams P, Jewell D: MicroRNAs and other tiny endogenous RNAs in C. elegans. Curr Biol 2003, I 3:807-818.

28. Grad Y, Aach J, Hayes G, Reinhart B, Church G, Ruvkun G, Kim J: Computational and experimental identification of $C$. elegans microRNAs. Mol Cell 2003, I I: | 253-I263.

29. Bieri T, Blasiar D, Ozersky P, Antoshechkin I, Bastiani C, Canaran P, Chan J, Chen N, Chen WJ, Davis P, Fiedler TJ, Girard L, Han M, Harris TW, Kishore R, Lee R, McKay S, Müller HM, Nakamura C, Petcherski A, Rangarajan A, Rogers A, Schindelman G, Schwarz EM, Spooner W, Tuli MA, Van Auken K, Wang D, Wang X, Williams G, Durbin R, Stein LD, Sternberg PW, Spieth J: WormBase: new content and better access. Nucleic Acids Res 2007:D506-10.

30. Kasprzyk A, Keefe D, Smedley D, London D, Spooner W, Melsopp C, Hammond M, Rocca-Serra P, Cox T, Birney E: EnsMart: A Generic System for Fast and Flexible Access to Biological Data. Genome Res 2004, 14:160-169.

31. Pavesi G, Mauri G, Pesole G: An algorithm for finding signals of unknown length in DNA sequences. Bioinformatics 2001, I 7:S207-S2 |4.

32. Pavesi G, Mereghetti P, Zambelli F, Stefani M, Mauri G, Pesole G: MoD Tools: regulatory motif discovery in nucleotide sequences from co-regulated or homologous genes. Nucleic Acids Res 2006, 34:W566-W570.

33. Tompa M, Li N, Bailey T, Church GM, De Moor B, Eskin E, Favorov A, Frith MC, Fu Y, Kent WJ, Makeev VJ, Mironov AA, Noble WS Pavesi G, Pesole G, Regnier M, Simonis N, Sinha S, Thijs G, van Helden J, Vandenbogaert M, Weng Z, Workman C, Ye C, Zhu Z: Assessing computational tools for the discovery of transcription factor binding sites. Nat Biotechnol 2005, 23:137-|44.

34. Bailey T, Williams N, Misleh C, Li W: MEME: discovering and analyzing DNA and protein sequence motifs. Nucleic Acids Res 2006, 34:W369-373.

35. Lawrence C, Altschul S, Boguski M, Liu J, Neuwald A, Wootton J: Detecting subtle sequence signals: a Gibbs sampling strategy for multiple alignment. Science 1993, 262:208-2 I4

36. Thompson W, Rouchka E, Lawrence C: Gibbs Recursive Sampler: finding transcription factor binding sites. Nucleic Acids Res 2003, 3 I:3580-3585

37. Chenna R, Sugawara H, Koike T, Lopez R, Gibson TJ, Higgins DG, Thompson JD: Multiple sequence alignment with the Clustal series of programs. Nucleic Acids Res 2003, 3 I:3497-3500.

38. Kankainen M, Pehkonen P, Rosenstöm P, Törönen P, Wong G, Holm L: POXO: a web-enabled tool series to discover transcription factor binding sites. Nucleic Acids Res 34:534-W540.

39. Crooks G, Hon G, Chandonia J, Brenner S: WebLogo: A sequence logo generator. Genome Res 2004, I 4: I I88- I I90.

40. Karolchik D, Kuhn RM, Baertsch R, Barber GP, Clawson H, Diekhans M, Giardine B, Harte RA, Hinrichs AS, Hsu F, Miller W, Pedersen JS, Pohl A, Raney BJ, Rhead B, Rosenbloom KR, Smith KE, Stanke M, Thakkapallayil A, Trumbower H, Wang T, Zweig AS, Haussler D, Kent WJ: The UCSC Genome Browser Database: 2008 update. Nucleic Acids Res 2008, 36:D773-9 [http://genome.ucsc.edu/ ].

4I. Brenner S: The genetics of Caenorhabditis elegans. Genetics 1974, 77:71-94.

42. Saeed Al, Sharov V, White J, Li J, Liang W, Bhagabati N, Braisted J, Klapa M, Currier T, Thiagarajan M, Sturn A, Snuffin M, Rezantsev A Popov D, Ryltsov A, Kostukovich E, Borisovsky I, Liu Z, Vinsavich A, Trush V, Quackenbush J: TM4: a free, open-source system for microarray data management and analysis. Biotechniques 2003, 34(2):374-378.
Publish with Biomed Central and every scientist can read your work free of charge

"BioMed Central will be the most significant development for disseminating the results of biomedical research in our lifetime. "

Sir Paul Nurse, Cancer Research UK

Your research papers will be:

- available free of charge to the entire biomedical community

- peer reviewed and published immediately upon acceptance

- cited in PubMed and archived on PubMed Central

- yours - you keep the copyright

Submit your manuscript here:

http://www.biomedcentral.com/info/publishing_adv.asp
BiolMedcentral 